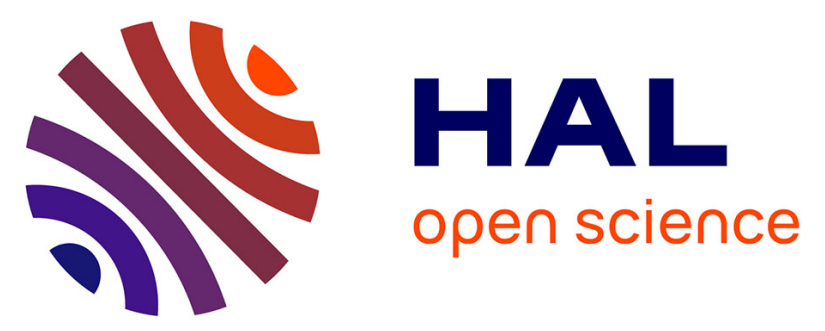

\title{
Une table en bronze inscrite d'Arles: hommages publics et pratiques testamentaires chez les notables d'ascendance italienne
}

\author{
Monique Dondin-Payre
}

\section{> To cite this version:}

Monique Dondin-Payre. Une table en bronze inscrite d'Arles: hommages publics et pratiques testamentaires chez les notables d'ascendance italienne. Gallia - Fouilles et monuments archéologiques en France métropolitaine, 1992, 49, pp.81-87. 10.3406/galia.1992.2930 . hal-01913146

\author{
HAL Id: hal-01913146 \\ https://hal.science/hal-01913146
}

Submitted on 19 Jan 2020

HAL is a multi-disciplinary open access archive for the deposit and dissemination of scientific research documents, whether they are published or not. The documents may come from teaching and research institutions in France or abroad, or from public or private research centers.
L'archive ouverte pluridisciplinaire HAL, est destinée au dépôt et à la diffusion de documents scientifiques de niveau recherche, publiés ou non, émanant des établissements d'enseignement et de recherche français ou étrangers, des laboratoires publics ou privés.

\section{(1) (1) $\$$}

Distributed under a Creative Commons Attribution - NonCommercial - NoDerivatives $\mid 4.0$ 


\title{
Une table en bronze inscrite d'Arles : hommages publics et pratiques testamentaires chez les notables d'ascendance italienne
}

\author{
par Monique DONDIN-PAYRE*
}

III Une table de bronze récemment découverte en Arles porte une dédicace à l'édile A. Pompeius Pius, fils C. Mlus, de la tribu Sabatina, élevée, à la demande testamentaire de sa mère, Kareia Ingenua, par son héritier, A. Pompeius Amomus. L'onomastique des protagonistes révèle leur origine : italienne, du nord-nord-est de 1 (Fururie, pour Pius dont la tribu Sabatina est caractéristique, et pour sa mère dont le gentilice est spécifique de cette région. Elle porte donc trace d'un noyau italien qui participa à la fondation d'Arles. La formulation des dispositions testamentaires indique que la mère de Pompeius eut recours à la procédure du fidéicommis pour faire honorer, par une statue sans doute, son fils, édile, décédé. Les frais devaient être prélevés sur le legs fait à son affranchi Pompeius Amomus, chargé d'exécuter sa volonté. La date proposée est le milieu du ${ }^{\text {er }} \mathrm{s}$.

A bronze tablet, recently discovered in Arles, bears a dedication to an aedile, A. Pompeius Pius, the son of Aulus, from the Sabatina tribe. This tablet was put up by A. Pompeius Amomus, the heir to Kareia Ingenua, Pius's mother, at her request expressed in her will. The origin of the protagonists is revealed by the onomastics: Italian from the North-North-East of Etruria for Pius's Sabatina tribe is characteristic of this region and his mother's gentilicium is also typical of this area. This onomastics refers to an Italian small group which took part in the foundation of Arles. The wording of the testamentary provisions suggests that Pompeius's mother resorted to the trust procedure to have her dead son, an aedile, honored, maybe with a statue. The expenses were to be drawn out from the legacy she had left her freed slave, Pompeius Amomus, responsible for carrying out her will. This tablet is likely to date from the middle of the 1st century.

Mots clés : table de bronze, dédicace, édile, tribu, colonie vétérane, affranchi, testament, héritier, fidéicommis, statue, Pompei, immigration, réemploi, Arles.

* C.VRS, Paris. 
Il y a un peu plus de dix ans, Jean Piton, membre du Laboratoire d'archéologie des musées d'Arles, mettait au jour, en creusant un trou dans son jardin pour y planter un arbre, une plaque de bronze inscrite, en excellent état de conservation (fig. 1 et 2); il la déposa aux musées d'Arles et en assura lui-même récemment la publication ${ }^{1}$. Ce document intéressant justifie une étude plus développée.

La découverte eut lieu à Trinquetaille, sur la rive droite du Rhône, et plus précisément à La Pointe (quartier de l'lle des Sables), dans une zone signalée depuis longtemps comme site d'une importante nécropole, insérée dans un habitat suburbain, mais aujourd'hui si densément occupée qu'on ne put y effectuer les fouilles qui démêleraient l'un de l'autre ${ }^{2}$.

La plaque de bronze, de grande taille $(80,5 \times 55,4 \times 1,5 \mathrm{~cm})$, est bordée sur sa face principale d'une rainure tracée à une distance de 0,5 à $2 \mathrm{~cm}$ du bord actuel; elle est inscrite d'une dédicace de six lignes mise en page avec soin pour occuper la partie supérieure, et gravée en majuscules bien formées (lettres de 3,8 à $4.8 \mathrm{~cm}$ ). Le nom du bénéficiaire est mis en valeur par des caractères plus gros (lettres de $5,5 \mathrm{~cm})$; certaines lettres sont plus hautes que les autres (ligne 1 : I de Pompeio, $6,8 \mathrm{~cm}$; lignes 3 et 5 : $\mathrm{K}, 5,3 \mathrm{~cm}$ et $4,5 \mathrm{~cm})$ et le tracé d'une même lettre peut varier (ligne 3 : la barre verticale du $\mathrm{K}$ est plus haute que les branches; ligne 5 , c'est l'inverse). Les mots sont séparés par des signes en forme de $\mathrm{V}$ ouvert, et, ligne 4, par des hederae aux tiges et pointes très développées.

Une version succincte de cette étude a étè présentée au colloque sur l'épigraphie rupestre qui s'est tenu à Rome en octobre 1989.

1 Découverte le 24 mars 1977, la plaque a été enregistrée au Musée sous le $n^{0}$ TRI 77 HS 01. Elle a été publiée dans C. Sintès dir., Du nouveau sur l'Arles antique, Revue d'Arles 1, catalogue d'exposition, mai-novembre 1987, Arles, 1987, p. 110-111 avec photographie en couleur. Je remercie très vivement M. J.-M. Rouquette, conservateur en chef des Musées d'Arles, qui a autorisé la publication des clichés et M. Cl. Sintès, conservateur aux Musées d'Arles, qui m'a fourni avec beaucoup d'obligeance toutes les précisions et les échantillons demandés.

2 Sur les nécropoles dont l'une fut vue aux $\mathrm{xvII}^{\mathrm{e}}$ et xviri $^{\mathrm{e}}$ s. lors de basses eaux, cf. L. A. Constans, Arles antique, Paris, 1921, p. 371-372.
Le texte se lit aisément : $A(u l o)$ Pompeio A(uli) f(ilio) / Sabat(ina tribu) Pio aed(ili) / Kareia Sex(ti) f(ilia) Ingenua / mater l(estamento) f(ieri) r(ogauit). I A(ulus) Kareius Amomus / h(eres) p(onendum) c(urauit) l(oco) d(ato) d(ecreto) d(ecurionum $)^{3}$.

$\mathrm{Au}$ dos, en caractères du mème type $(3,5$ à $4,4 \mathrm{~cm}$, sauf, ligne 1 , le $\mathrm{T}$ final de $5,2 \mathrm{~cm})$ :

Testamento / sibi fieri iussit.

L'élucidation du détail des informations du texte est délicate, bien que sa teneur générale paraisse très claire : l'édile A. Pompeius Pius est, conformément à une disposition testamentaire de sa mère, Kareia Ingenua, exécutée par l'héritier A. Kareius Amomus, honoré d'une façon qui n'est pas spécifiée.

L'onomastique, simple, est révélatrice du statut social de chacun des protagonistes. On énumère pour l'édile A. Pompeius sa nomenclature complète, selon les normes de la lex Iulia municipalis ${ }^{1}$. Le gentilice Pompeius, banal en Narbonnaise, est brillamment représenté en Arles par les Pompeii Paulini : leur fortune fut assurée par P. Pompeius, préfet de l'annone, dont une fille épousa Sénèque, ils accédèrent au sénat au milieu du $\mathrm{e}^{\mathrm{er}} \mathrm{s}$. après J.-C. ${ }^{5}$. Mais,

3 Pour la première publication, on se reportera loc. cit.. p. 111 et $A E, 1988,859$.

4 Une nomenclature du type de celle de Pompeius est en usage en Varbonnaise dès une époque haute ( $\mathrm{I}^{\mathrm{er}} \mathrm{s}$. avant J.-C.) : E. Demougeot, Stèles funéraires d'une nécropole de Lattes, Revue archéologique de Narbonnaise, 5, 1972, p. 55, 58.

5 Le gentilice Pompeius est très commun en Narbonnaise comme dans la plupart des autres provinces; il est, proportionnellement, peu représenté en Arles : une douzaine de cas $(C I L$ XII, 732, 736, 762, 806, 861 à 864, 871, 956, 5815; $I L G N, 133,134)$ et très peu de Pompeianus ( 701 et 807 au $C I L)$. Bien qu'il se soit répandu dans cette province principalement grâce ḋ l'extension des clientèles pompéiennes (E. BaDian, Foreign clientelae (264-70 B.C.), Oxford, 1958, p. 251-257, 263, $267 \mathrm{sq.:}$ - voir aussi Y. Burnand, Domitii Aquenses. Une famille de chevaliers romains de la région d'Aix-en-Provence, mausolee el domaine, $5^{\prime \prime}$ suppl. à la Revue archéologique de Narbonnaise, Paris, 1975), il ne doit pas être rattaché systématiquement à Pompée le Grand; contra J. Piton, La Pointe, Revue d'Arles 1, p. 111 et M. Christol, Notes d'Épigraphie Varbonnaise, Études sur Pézenas et sa région, VI, 2, 1975, p. 3. Sur les Pompeii Paulini : PIR P479 et 480 ; le père, chevalier et préfet de l'annone, beau-père de Sénèque : Y. Burnand, Senatores ex provinciis Galliarum orti, Epigrafia e ordine senatorio, Il, Tituli 5, Rome. 1982, p. 413 (notice), 435, et C. CasTIL..o, Los senatores Béticos - relaciones familiares y sociales, ibid., p. 490 (ses relations avec Sénèque); le fils, consul et légat de Germanie : Plinf, IIN, XXXII, 143, notice très complète par W. Eck, Die Statthalter der germanischen Provinzen vom 1.-3. Jahr., Epigraphische Studien 14, Cologne-Bonn, 1985, p. 120-122, no 7. R. Srme, Tacilus, 2, Oxford, 1958, p. 591 : le gouvernement de Germanie présenté à la lumière de l'interven- 


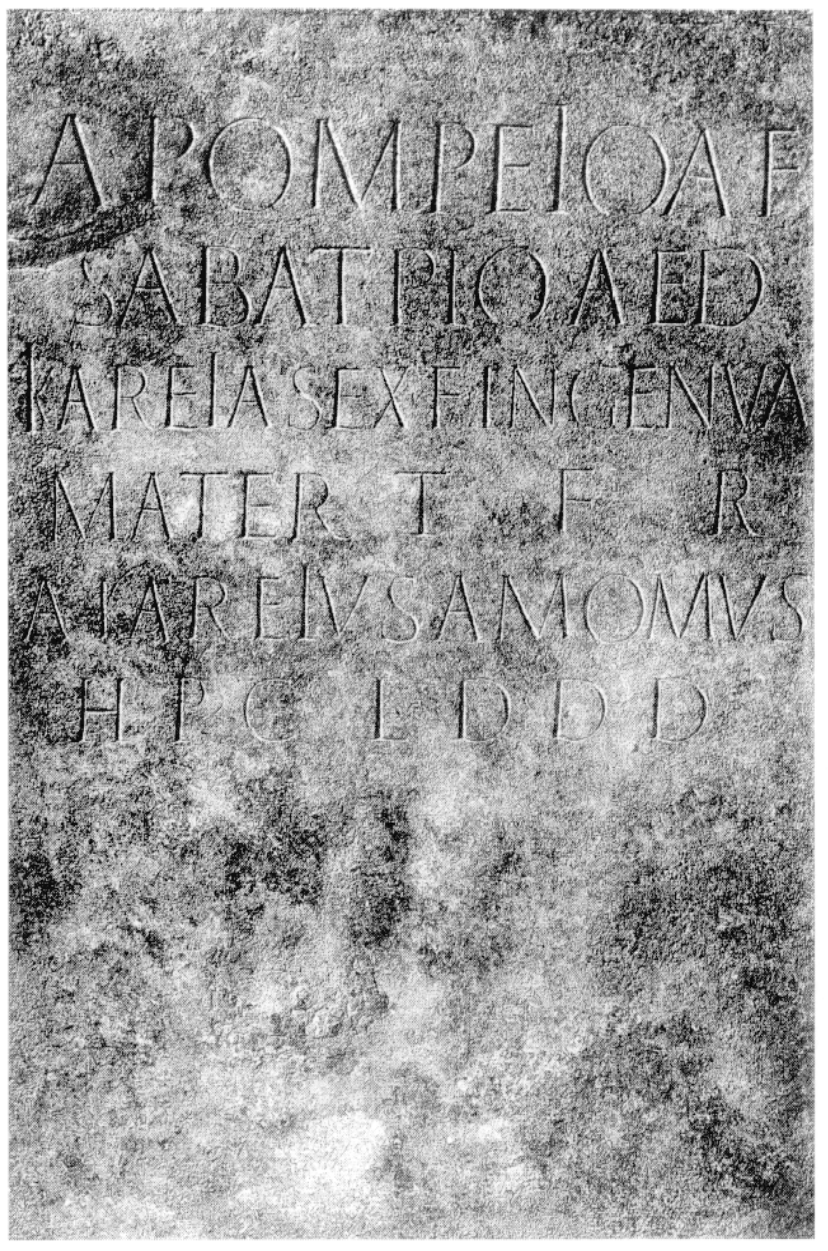

Fig. 1 - Recto de la plaque en bronze. Cliché Michel Lacanaud, Musées d'Arles.

combiné avec la tribu Sabatina, il oriente vers une origine italienne ${ }^{6}$ car cette tribu, qui n'est ni celle d'Arles (la Teretina) ni celle d'aucune ville en Gaule (à l'exception de Mantoue en Cisalpine), est en

tion de Sénèque, maître de l'empire à la place de Véron (idée reprise dans Colonial elites - Rome, Spain and the Americas, Oxford, 1958 , p. 8, 18-20), 603 : ses relations familiales avec Sénèque, 620 : ses origines arélates.

6 Sur la tribu Sabatina, voir L. R. TAYLOR, The Voting Districts of the Roman Republic - The 35 Urban and Rural Tribes, Papers and Monographs of the American Academy in Rome, 20, Rome, 1960, p. 48-49, 85-89. 115-117; J. W. Kuвiтsснғк, Imperium Romanum tributim discriptum, Prague, 1889. p. 272 (voir fig. 3). Elle est citée deux fois en Narbonnaise : sur un cippe de Narbonne, dans la liste des ayants droit aux tombeaux (CIL XII, 5138, ligne 6: [ .../o Licinio D.f.Sab.Primo tutori) et sur une pierre de Carpentras (CIL XII, 1209) où sa mention parut si suspecte à Hirschfeld qu'il l'invoque comme argument pour classer le texte parmi les faux (tribus ab his regionibus aliena), avec le mot uiridarium et une ligature insolite.

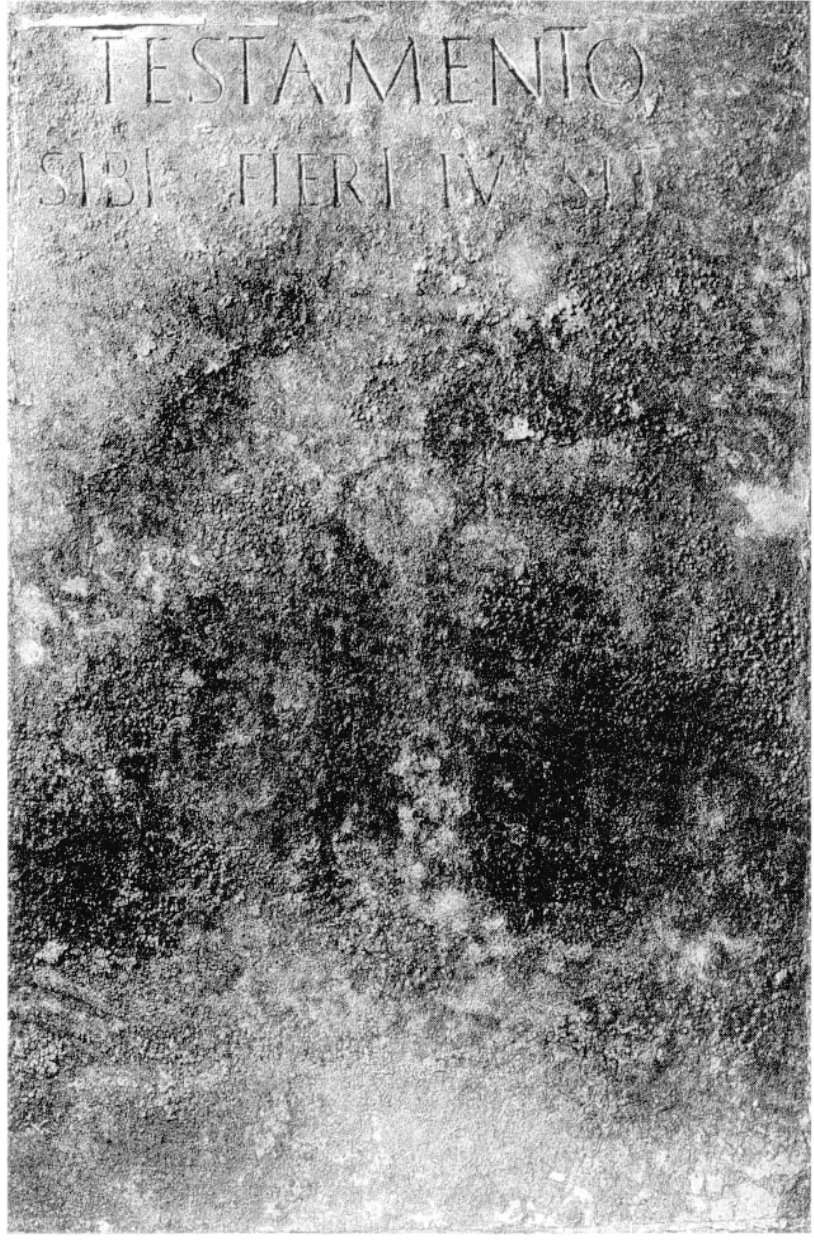

Fig. 2 - Verso de la plaque en bronze.

Les traces de la bande de fixation sont visibles le long des quatre bords. Cliché Michel Lacanaud, Musées d'Arles.

revanche caractéristique de la regio VII : Pompeius est donc très probablement issu d'une famille d'Italie installée en Arles. Le processus n'a rien pour étonner : il a déjà été établi que, contrairement aux allégations de Mommsen et de Hirschfeld qui jugeaient que César n'avait établi ses vétérans qu'en Italie, le noyau des fondateurs de la colonie arélate compre-

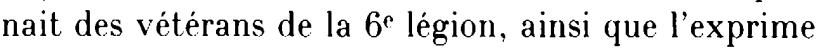
le terme Sextani inclus dans la titulature (colonia Iulia Paterna Arelale Sextanorum) ${ }^{\text {? }}$. Or cette légion, que L.-A. Constans pensait avoir été levée à la limite du Samnium et du Latium, puisque les huit cités inscrites dans la Teretina (qui n'est pas celle de César, fondateur de la colonie d'Arles), y sont situées, semble avoir été partiellement recrutée au

7 O. Hirschfeld, CIL XII, p. 83. Contra L. A. Constans, op. cit., p. 53, 56-58. Voir M. Christol, op. cil., p. 3, 4 pour l'exposé de la discussion et la bibliographie antérieure. 


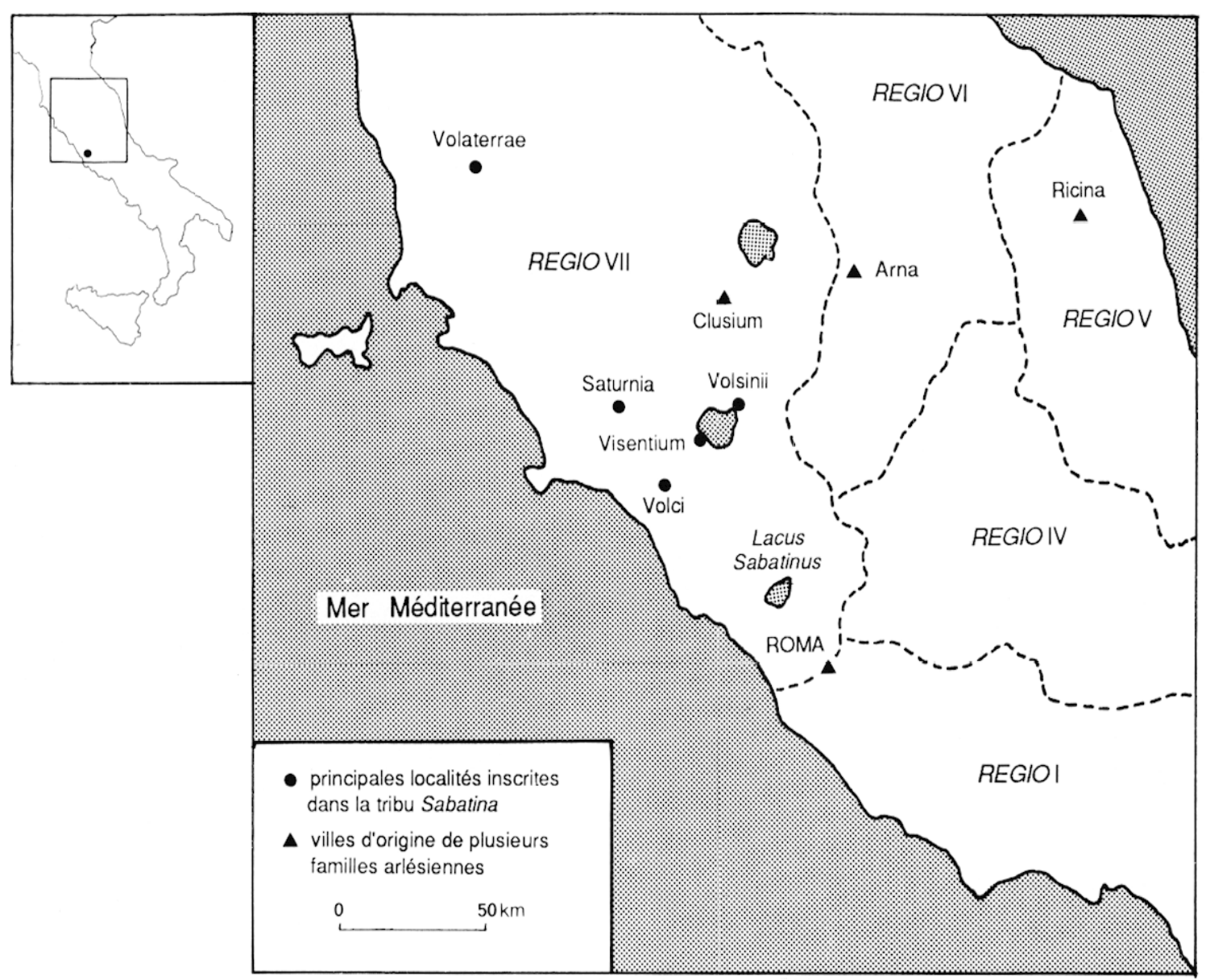

Fig. 3 - Carte de répartition de la tribu Sabatina. La tribu Sabatina est très peu répandue dans l'empire romain. Créee en 387 pour répartir les habitants du territoire de Veii nouvellement annexé (T.L., VI, 5, 8, : Tribus quattuor ex nouis ciuibus additae Stellatina Tromentina Sabatina Arniensis), elle s'étendait à l'origine au nord du lac Sabatinus d'où elle tirait son nom (Festus, 464L). Après la guerre sociale, son territoire fut fractionné puisqu'on lui rattacha la région de Volci et surtout celle de Volaterrae, très peuplée. Son effectif s'accrut quand on lui affecta, à l'initiative de Pompée, les Transpadans de Mantoue. Mis à part cette zone, la tribu Sabatina resta limitée à la regio VII et à l'Étrurie (voir note 6 ; les occurrences récentes le confirment : Annexe, p. 87).

nord-nord-est de l'Étrurie, dans la zone où est répandue la tribu Sabatina, ou à proximité immédiate. Plusieurs familles arélates proviennent de cette partie de l'Italie : les Annii Camarles que H.-G. Pflaum avait rattachés à la ville de Clusium, en Étrurie, les porteurs du nom Viblatro en lesquels $M$. Christol a reconnu des Ombriens d'Arna, le sévir augustal Aelanius qu'il rapproche des homonymes de Ricina, dans le Picenum, peut-être aussi les Saenii ou les Praecilii ${ }^{8}$. L'appartenance de A. Pompeius Pius à la tribu Sabatina apporte un nouvel argument en faveur de l'hypothèse d'un noyau italien, issu du

8 II.-G. Pri.aum, Une famille arlésienne à la fin du $\mathrm{r}^{\mathrm{er}} \mathrm{s}$. et au $\mathrm{II}^{\mathrm{e}} \mathrm{s}$. de notre ère, Bulletin de la Société nationale des Antiquaires de France, 1970, p. 265-272; - M. Christol, op. cit., p. 5, 6 avec la bibliographie antérieure. nord - nord-est de l'Étrurie, qui contribua à la fondation d'Arles (fig. 3). Mieux encore, la mère de Pompeius, Kareia, était originaire de la même région : une localité d'Étrurie est ainsi dénommée, et le gentilice y est attesté ${ }^{9}$. La précision de la filiation, Sex. $f$. , inaccoutumée pour une femme, exprime la fierté d'appartenir à une souche purement italienne. Le

9 J'avais pensé que la graphie Kareia, au lieu de Careia, s'appliquait à une famille gauloise romanisée : il n'y a aucun Careius/Kareius en Arles, mais une douzaine dans la province (6 au CIL XII, autant dans E. Espérandieu, Inscriptions latines de Gaule (Narbonnaise), Paris, 1929, qui à propos de L. Kareius Vitalis (184, Orange) commentait : "Le gentilice Kareius est assez fréquent en Narbonnaise»). Je remerce MM. les professeurs L. Gasperini et S. Panciera de m'avoir signalé les homonymies qui orientent vers une origine étrusque. Karius/Karia : CIL XI, 474. 
troisième personnage, A. Kareius Amomus, ne donne, lui, que des informations minimales : ses tria nomina, sans préciser ni filiation ni tribu. Ce laconisme, insolite après les développements onomastiques précédents, l'homonymie avec la mère, et le cognomen grécisant révèlent très probablement ce sur quoi Amomus ne souhaite pas attirer l'attention : sa qualité de libertus ${ }^{10}$. A. Kareius Amomus a été affranchi par Kareia Ingenua; il n'a pas adopté le prénom du père de sa domina, Sextus, mais Aulus, celui du mari et du fils.

Il est le pivot du texte puisque c'est à lui que Kareia Ingenua confia, par testament - ligne 4: l(estamento) f(ieri) r(ogauil) - le soin d'honorer son fils, édile. Bien que le cas de figure soit banal, plusieurs points sont à préciser : la procédure légale employée, la nature de l'intervention de Kareius Amomus et du monument qu'il fit élever à A. Pompeius Pius.

Kareia Ingenua a, par testament, institué héritier A. Kareius Amomus, son affranchi : la pratique est courante, mais il ne faut pas en déduire systématiquement qu'Amomus est unique heres et, que, par conséquent, A. Pompeius est mort et bénéficie d'un hommage funéraire. Ce point mérite débat. Pour témoigner son estime à son affranchi. Kareia lui a légué une partie de sa fortune (ligne 6 : heres), en disposant du reste à son gré, en faveur de son fils seul, s'il est encore vivant, ou de plusieurs autres bénéficiaires. Elle assortit ce legs d'une clause conditionnelle, selon la procédure du fidéicommis, en vigueur depuis Auguste et à laquelle sa souplesse valut immédiatement une grande faveur ${ }^{11}$. Que cette

10 Amomus (Thesaurus Linguae Latinae, 1, Leipzig, 1900, col. 1967) pourrait peut-être être rapproché de Pline, IIN, XII, 48-49: amomum. plante aromatique dont le dérivé adjectif est amomis; il est caractéristique du milieu servile et affranchi. Il n'est pas attesté au CIL XII, mais figure sept fois au CIL VI : un citoyen romain (2686: Q. Modius Q. f. Pollia Amomus, soldat de la $8^{\circ}$ cohorte prétorienne), deux fois à propos d'hommes de statut indéterminé $(15620$ et 29428$)$ et quatre pour des esclaves et affranchis $(4257,5875,15696$, 34302). Voir II. Sot. In, Die Griechischen Personennamen in Rom - Ein Namenbuch, Berlin - New York, 1982, p. 728, cf. p. 379.

11 MM. Moreau et J. Stern m'ont apporté une grande aide pour la compréhension de cet aspect du problème; je les remercie infiniment pour leurs informations et leurs avis. L'ouvrage le plus récent sur le fidéicommis: D. Johnston, The Roman Law of Trusts, Oxford, 1988, donne un bon aperçu général sur ces dispositions apparues sous Auguste et sur leur évolution. Pour des études plus techniques : M. Amelotri, $I I$ leslamento romano altraveso le prassi documentale, 1 : Le forme classiche di testamento, Florence, 1966, p. 130-148: B. Biondi, Successione testamentaria e donazioni, Milan, 1955, p. 286 et $475 s q . ;-$ A. Guarino, Diritto privato romano, disposition ait été utilisée est prouvé par la formule, peu fréquente en épigraphie, mais non inconnue, fieri rogauit, dont le verbe rogare est cité par les sources juridiques comme un de ceux qui introduisent une disposition de fidéicommis ${ }^{12}$. Le mot n'a pas été employé au hasard puisque, au dos de la table, on a préféré fieri iussit. Pour hériter, Kareius Amomus devait donc rendre à $\mathrm{A}$. Pompeius Pius un hommage précis, qui n'est pas décrit sur la plaque. Les circonstances de la découverte ne permettent de savoir ni dans quel contexte archéologique elle s'inscrivait ni quel monument elle accompagnait ${ }^{13}$. Mais on peut, avec une grande vraisemblance, proposer une statue, pour trois raisons : à cause du verbe ponere, qui convient à une statue et non à un monument (facere), parce que les décurions ont accordé un emplacement public, pratique attestée fréquemment pour des statues de personnages officiels ${ }^{14}$, mais jamais adoptée pour des monuments sans qu'un funus publicum l'accompagne, ce qui serait excessif pour un édile; enfin, le juriste Pomponius prévoit un cas de figure exactement similaire au nôtre : "Titius si statuas in municipio posuerit, heres esto. Si paratus est ponere, sed locus a municipibus ei non datur, Sabinus Proculus heredem eum fore, et in legato idem iuris esse dicunt " (8 Ad Sabinum, Dig. 35, 1, 14) ${ }^{15}$. Comme

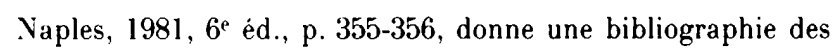
clauses conditionnelles testamentaires; - P. Vocı, Diritto ereditario romano, 2, Milan, 1963, p. 223 sq., 596-598.

12 Rogare: Vocabularium iurisprudentiae Romanae, 5 , Berlin, 1939, s.v. rogo, col. 216-222, surtout col. 217 B et 218 E. Exemples d'occurrences épigraphiques : CIL I, 1019= CEL 68 (quod rogari ut faceret monumentum mihi), CIL V, $5873,5898,6896 ;$ IX, $4519=I L S 6545 ; \mathrm{XII}, 2928$. L'exposé de la valeur de rogare pour les fidéicommis: Gaius, II, 249 Verba autem fideicommissorum haec recte maxime in usu esse uidentur : peto, rogo, uolo, fideicommilto, quae proinde firma singula sunt atque si omnia in unum congesta sint ("Voici les mots valables pour les fidéicommis, qui sont d'usage le plus courant 'je demande', 'je sollicite', 'je souhaite', 'je confie'; ils sont aussi efficaces individuellement que s'ils sont employés ensemble").

13 Voir supra note 2.

14 On peut, parmi d'autres exemples, comparer la plaque de Pompeius à la base de Gauia M. fil. Marciana, de Pouzzoles (CIL X, 1784), qui énumère les honneurs qui lui furent décernés après sa mort, notamment trois statues: R. Preux, L'inscription funéraire de Gauia Marciana et le décret municipal en son honneur. Étude sur le milieu des notables de Pouzzoles à la fin $d u{ }_{11}{ }^{\mathrm{e}} \mathrm{s}$. ap. J.-C., thèse de Doctorat de $3^{\text {e }}$ cycle, Paris, 1968, dactyl., surtout p. 69-71 à propos des statues et p. 90,91 pour l'emplacement public.

15 "Que Titius soit mon héritier, à condition d'avoir, auparavant, fait placer des statues dans le municipe. S'il est disposé à les ériger, mais que le lieu ne lui est pas concédé par les citoyens du municipe, d'après Sabinus et Proculus, il sera 
Kareius, Titius devenait héritier s'il élevait des statues en l'honneur du testataire; pour ce faire, il devait solliciter de la curie la cession d'un terrain; si elle était refusée, soit, comme Titius, l'héritier était libéré de son obligation, soit, et ce pouvait être le cas pour Kareius, il devait acheter le terrain grâce à la somme léguée.

La teneur du document est donc clarifiée. Reste à régler la question de savoir si l'édile est honoré à titre posthume ou de son vivant. Les circonstances de la découverte ne permettent pas de trancher, puisque les fouilles n'ont pu être menées et que la nécropole attestée dans la zone est tardive. Les données juridiques non plus : rien n'empêche que A. Kareius Amomus soit cohéritier, pour la part dont la mère peut disposer sans léser son fils; Kareia Ingenua aurait pu faire à la communauté un legs si important que l'ordo aurait consenti envers un de ses membres jeunes, l'édile Pompeius Pius, un honneur, la cession d'un terrain public pour lui ériger une statue, excessif par rapport à son statut; A. Pompeius Pius aurait pu ensuite poursuivre sa carrière et accéder, éventuellement, à des fonctions supérieures. Mais il faut considérer les usages que mettent en évidence les inscriptions; ils éloignent de cette reconstruction, valable par ailleurs : les magistrats vivants ne sont honorés ainsi que de façon exceptionnelle, s'ils occupent une place remarquable; or Pompeius n'est qu'édile, sans autre caractéristique à notre connaissance. Il est donc très probable que Pompeius est mort jeune, avant sa mère; Kareia force en quelque sorte le destin en lui faisant ériger, sur un terrain public, un monument qui dépasse en splendeur ce à quoi sa gloire personnelle lod aurait donné droit. Elle perpétue ainsi la double mémoire d'une famille, celle des Pompeii, sans doute éteinte, et la sienne : les deux gentilices sont cités. Par là, ces deux souches italiennes renommées en Arles, et riches puisque ce legs qui implique une grosse mise de fonds ne reflète qu'une partie de leur fortune, compensaient en quelque sorte une injustice : le rang d'édile atteint par le fils prématurément disparu ne correspondait pas à leur situation sociale prééminente.

héritier et, dans le cas d'un legs, la situation juridique est la mème". Ce texte, qui souligne l'unanimité des juristes (Sabinus et Proculus sont. les chefs de deux écoles rivales) sur les dispositions concernant l'héritage, ne s'applique pas exactement au fidéicommis mais à l'héritage conditionnel, une procédure très voisine.
La présentation matérielle de la plaque est un peu insolite : elle porte au revers deux lignes d'une graphie aussi soignée que celle de l'avers; la mention testamento sibi fieri iussit signifie à première vue que Kareia a joint à la célébration de son fils une disposition plus modeste qui la concernait personnellement, dans laquelle son affranchi n'était pas obligatoirement impliqué. Mais on ne peut imaginer la plaque exposée de sorte que ses deux faces soient lisibles. La présence d'un liséré de métal au revers donne la solution; il ne s'agit pas d'une finition ornementale, destinée à former une sorte de cadre, mais des restes de la bande de soudure, qui maintenait la plaque contre son support ${ }^{16}$. Le revers n'était donc pas destiné à être lu : la plaque de bronze était appliquée sur la base de la statue de Pompeius Pius, et le texte de deux lignes témoigne d'un premier état : gravée pour un monument funéraire, elle fut jugée, pour une raison indéterminée, inadéquate. Cependant, ètant donné sa qualité, elle ne fut pas fondue, mais retournée et réutilisée par le bronzier. Ce réemploi témoigne-t-il d'un souci d'économie de la part de l'exécuteur testamentaire qui ne voulait pas trop écorner son legs en engageant des dépenses importantes? Ou de la nécessité d'exécution rapide des prescriptions de Kareia pour que les clauses soient valables? Il est impossible de trancher.

La date du texte peut être fixée à partir de plusieurs critères : en premier lieu à partir d'un indice extérieur, la présence d'hederae; leur substitution à la ponctuation en $\mathrm{V}$ (ligne 4) ne permet pas de fixer la date de fabrication du document au II $^{\mathbf{e}}$ s., époque à laquelle se répandirent, sur la pierre, ces signes apparus sous Tibère à Rome : les coutumes de la graphie sur bronze peuvent être différentes de celles des lapicides. Plusieurs éléments permettent d'avancer une datation plus haute, vers le milieu du $\mathrm{I}^{\mathrm{er}} \mathrm{s}$. : la tribu, atypique, que des recensements répétés auraient effacée au profit de la tribu Teretina, celle d'Arles, le rapprochement avec les célèbres Pompeii, chevaliers puis sénateurs, du milieu du I $^{e r}$ s., dont il convient de ne pas trop éloigner ces Pompeii plus modestes; enfin, la permanence, dans la population arlésienne, d'un noyau italien, refermé sur lui-mème,

16 Je remercie très vivement M. le Professeur M. Mayer de mavoir indiqué l'usage de la bande de métal, que j'avais prise pour une décoration de la plaque; il a bien voulu faire procéder, à l'Université de Barcelone, à une analyse qui a confirmé son hypothèse et $m$ 'a signalè l'existence d'un parallèle à Ampurias. 
ne permet pas de s’éloigner beaucoup des débuts de la colonie. La paléographie, les formules onomastiques, l'usage du fidéicommis confirment la date de ce document qui, sous un aspect anodin, atteste la part prise dans la fondation d'Arles par des éléments de population venus d'Italie : création césảrienne ${ }^{17}$,

17 L. A. Constans, op. cit., p. 51-55. Sur les fondations concomitantes d'Arles et de . Varbonne par César : SuÉrone, Tib.. IV, 2 : ad deducendas in Galliam colonias in quis Narbo destinée à surveiller Marseille dont la fidélité semblait douteuse, la colonie comportait un fort contingent italien, qui préserva longtemps sa spécificité.

\section{Monique Dondin-Payre} Chr. Gocoineau, Note sur la fondation de Lyon, Gallia, 44, 1986, p. 172-173.

\section{ANNEXE \\ LES MENTIONS RÉCENTES DE LA TRIBU SABATINA}

Presque toutes les mentions récentes de la tribu Sabatina renvoient à la regio VII : à Visentium (Année épigraphique (AE), 1962, 151 et 153, AE, 1974,329 repris dans $A E, 1980,428$ ì, à Saturnia $(A E, 1977,257$ et 1978,307$)$, ̀̀ Volaterrae et à sa région $(A E, 1982,328,355$ et 356 : Cecina $, A E, 1972$, 615: M. Petronius Vmbrinus citè à Altaleia, en Lycie-Pamphylie, est originaire de Volaterrae, cf. M. Torelli, Epigrafia e ordine senatorio. II, Tituli כ, Rome, 1982, p. 290).

Plusieurs soldats originaires de Mantoue sont attestés dans l'empire :

- à Rome : $A E, 1984,66$ : C. Petronius Aullus, Mantua, de la $2^{\text {e }}$ cohorte urbaine;

- et surtout en Germanie : CIL, III, 6979 repris dans $A E, 1981,719$; à Mongotiacum L. Sergius Valentinus, Mantua, de la 22e Primigenia (AE, 1982, 719); à Osterburken Q. Cornelius Seuerinus, de la même légion, sans origine citée $(A E, 1985,691)$; à Bonn, dans l'épitaphe fragmentaire d'un soldat de la $21^{\circledR}$ Rapax, la lettre B qui subsiste seule du nom de la tribu renvoie peut-être à la Sabatina $(A E, 1977,578)$; mais, étant donné la rareté de celle-ci, il faut sans doutc lui préférer la Fabia (plutôt que la P'oblilia).

Dans trois cas, la relation avec la regio VII ou avec Mantoue n'est pas exprimée :

- poưf C. Valerius Vindemitor cité dans une dédicace privée d'Antemnae (regio I) sans aucune précision qui permette d'établir son origo $(A E, 1978,67)$;

- pour L. Firminius Maximus et son frère honorés à Valence d'ornamenta municipaux et étrangers à la ville $(A E, 1976,393)$;

- pour M. Valerius Dotus, mort à Morsano (regio X près d'Aquilée) ( $A E, 1981,396)$.

$$
\text { M. D.-P. }
$$

